\title{
Validation of transit-time flowmetry for chronic measurements of regional blood flow in resting and exercising rats
}

S.L. Amaral and

L.C. Michelini
Departamento de Fisiologia e Biofísica, Instituto de Ciências Biomédicas, Universidade de São Paulo, 05508-900 São Paulo, SP, Brasil

\section{Correspondence}

L.C. Michelini

Departamento de Fisiologia

e Biofísica, ICB-USP

Av Prof. Lineu Prestes, 1524

05508-900 São Paulo, SP

Brasil

Fax: 55 (011) 818-7285 and

813-0845

E-mail: lisete@bmb.icb1.usp.br

Research supported by CNPq and

FAPESP. S.L. Amaral was the

recipient of a CNPq fellowship.

Received October 9, 1996

Accepted April 29, 1997

\begin{abstract}
The objective of the present study was to validate the transit-time technique for long-term measurements of iliac and renal blood flow in rats. Flow measured with ultrasonic probes was confirmed ex vivo using excised arteries perfused at varying flow rates. An implanted 1$\mathrm{mm}$ probe reproduced with accuracy different patterns of flow relative to pressure in freely moving rats and accurately quantitated the resting iliac flow value (on average $10.43 \pm 0.99 \mathrm{ml} / \mathrm{min}$ or $2.78 \pm 0.3 \mathrm{ml}$ $\min ^{-1} 100 \mathrm{~g}$ body weight ${ }^{-1}$ ). The measurements were stable over an experimental period of one week but were affected by probe size (resting flows were underestimated by $57 \%$ with a $2-\mathrm{mm}$ probe when compared with a $1-\mathrm{mm}$ probe) and by anesthesia (in the same rats, iliac flow was reduced by $50-60 \%$ when compared to the conscious state). Instantaneous changes of iliac and renal flow during exercise and recovery were accurately measured by the transit-time technique. Iliac flow increased instantaneously at the beginning of mild exercise (from $12.03 \pm 1.06$ to $25.55 \pm 3.89 \mathrm{ml} / \mathrm{min}$ at $15 \mathrm{~s}$ ) and showed a smaller increase when exercise intensity increased further, reaching a plateau of $38.43 \pm 1.92 \mathrm{ml} / \mathrm{min}$ at the 4 th $\min$ of moderate exercise intensity. In contrast, exercise-induced reduction of renal flow was smaller and slower, with $18 \%$ and $25 \%$ decreases at mild and moderate exercise intensities. Our data indicate that transit-time flowmetry is a reliable method for long-term and continuous measurements of regional blood flow at rest and can be used to quantitate the dynamic flow changes that characterize exercise and recovery.
\end{abstract}

\section{Introduction}

There is increasing interest in the continuous measurement of total and regional blood flow in conscious animals. Electromagnetic (1-7) and pulsed Doppler ultrasonic flow probes $(4,5,8-10)$ have been widely used for these measurements but they present some disadvantages. Electromagnetic
Key words

- Ultrasonic transit-time

- Iliac blood flow

- Renal blood flow

- Exercise

- Anesthesia

- Conscious rats 
ric blood flow. Perivascular transit-time probes measure blood flow by emitting a plane of ultrasonic sound that transits the cross section of the vessel in both the upstream and downstream directions. The difference between the integrated transit-times of accelerated (directed downstream) and decelerated (directed upstream) ultrasonic signals, that interact with the volume flow occurring within the vessel, is a measure of volume per minute ( $\mathrm{ml} / \mathrm{min}$ or $1 / \mathrm{min})$. Since the transit-time technique gives absolute flow, independently of vessel size, and is easily implantable due to the reduced dimensions of the apparatus, it is adequate for chronic studies. In the rat, transit-time flowmetry has been widely used to quantitate blood flow in gastric $(11,12)$, renal $(13,14)$, mesenteric $(13,15)$, carotid (13), and femoral (13) arteries and in the aorta $(13,15)$ but only under acute conditions.

The validation of transit-time probes has been done in large animals under a variety of experimental conditions $(3,7,16,17)$. In the rat, renal (14), mesenteric and aortic flows (15) were measured with Transonic probes and compared with known blood/saline flows obtained from pump-perfused circuits (15) or by clearance of p-aminohippuric acid (14). These data, obtained in acute experiments, have demonstrated the accuracy of the transit-time method for blood flow measurements. No information on the stability of chronic measurements by the transit-time probe is available for conscious freely moving rats.

All previous studies concerning regional blood flow changes induced by exercise in rats (18-21) were conducted using the microsphere technique, which only provides information about static but not dynamic flow behavior. There is no information about continuous measurements and/or time-course changes of regional blood flow during maintained exercise and recovery. Studies of continuous blood flow changes simultaneous to pressure/heart rate changes during exercise are also lacking. In order to standardize the transit-time flowmetry for recording regional blood flow in rats, the specific objectives of the present study were 1) to validate true flow values obtained in vitro with $1-\mathrm{mm}$ and 2-mm probes, 2) to determine the sensitivity of this technique in reproducing the instantaneous changes of flow simultaneously with pressure in freely moving rats, 3) to evaluate the accuracy of the 1-mm probe in recording sequentially the basal iliac flow (IF) after chronic implantation and 4) to describe the dynamic behavior of hindlimb and renal flow at rest, during exercise and recovery.

\section{Material and Methods}

\section{In vitro perivascular flow probe testing}

The flow probes were submitted to bench tests before each implantation to confirm their performance. The values of the test signal were high $(0.9 \pm 0.06$ to $1.02 \pm 0.05 \mathrm{~V})$ as recommended by the manufacturer and did not change throughout the experiment.

To assess the validity of 1-mm and 2-mm flow probe measurements over a wide range of flow values, a first series of experiments was conducted in vitro. The common carotid artery was removed from rats immediately after sacrifice under deep pentobarbital anesthesia and mounted in a circuit driven by a peristaltic (LKB, Bromma, 2115, multiperpex) or syringe pump (Orion, M 362). Transit-time ultrasonic probes (Transonic System, Ithaca, NY) were adapted around the vessel and an acoustic couplant was applied to fill the space between the carotid and transit-time probe and to provide adequate acoustic conduction. The circuit was filled with saline (transit-time flow measurements are independent of hematocrit) (14) and its extremity was opened into a graduated test tube to confirm the true flow. A wide range of known flow rates from 0 to $20-30 \mathrm{ml} / \mathrm{min}$ was passed through the artery and measured with the flowmeter (Transonic System, T 206 dual channel). 


\section{In vivo studies}

All surgical procedures and protocols used were in accordance with the Institutional Guidelines for Animal Experimentation.

Male Wistar Kyoto rats (250-300 g, 2-3 months old) were anesthetized with sodium pentobarbital $(50 \mathrm{mg} / \mathrm{kg}$, ip $)$. Two $1-\mathrm{cm}$ dorsal incisions were made in the skin overlying the midscapular and iliac regions, and a blunt hemostat was used to create a subcutaneous tunnel between them. A midline incision was performed in the abdominal wall and the left iliac artery just below the aortic bifurcation (hindlimb studies) or the left renal artery (renal blood flow studies) was exposed and isolated for probe placement. All fatty tissue was carefully removed from the isolated segment of the vessel to avoid obstruction of the ultrasonic signal. The 1-mm (or 2-mm in one of the protocols) transit-time ultrasonic flow probe (model 1RB) was placed on the cleared section of the vessel. The probe was sutured to the underlying musculature and the segment of the artery containing the probe was covered with Lyostypt (Braun, Melsungen, Germany) to maintain vessel/probe alignment and to inhibit fat infiltration. The probe cable was passed through a small incision made in the retroabdominal wall and tunneled subcutaneously to the dorsal midscapular region. The probe was tested for the presence of a good acoustic signal, and blood flow during anesthesia was recorded for a period of 10-20 min (Gould recorder, model 4000, Cleveland, OH). The abdominal incision was then sutured and the connector end was fixed to the skin. The rats were treated with 60,000 units $(0.1 \mathrm{ml}, \mathrm{im})$ of penicillin (Pentabiótico Veterinário Fontoura, São Paulo, SP, Brazil). To record blood pressure, a clear vinyl catheter ( $3 \mathrm{~cm}$ of \#10 tubing connected to $13 \mathrm{~cm}$ of \#90 tubing) was inserted into the right iliac artery through the femoral artery of ether-anesthetized rats. The external part of the cannula was tunneled subcutaneously through the midscapular re- gion. The rats were allowed to recover for a period of $24 \mathrm{~h}$.

\section{Experimental protocols}

Four groups of rats were studied. In group I, 10 conscious rats implanted with a 1-mm probe into the iliac artery were submitted to daily blood flow and heart rate (HR) measurements (40-60 min experimental sessions) for 5 days. The experimental sessions were conducted at the same time (about 8-10 a.m.). All behavioral activities (exploring, alert, rest, etc.) exhibited by the rats were annotated. Daily blood flow and heart rate values considered for each rat were those representing the respective means of all resting periods recorded. On the 5th day, the rats had the arterial cannula implanted and the recording session was repeated on the next day to assess the accuracy of the probe in detecting the possible patterns of iliac flow with respect to spontaneous pressure oscillations in conscious freely moving rats. The rats were then anesthetized with ether and the abdomen was reopened to expose the iliac artery plus the probe. The probe was calibrated for zero baseline function blood flow using the internal calibration system of the flowmeter. Under conditions of zero blood flow in the calibration meter, the normal ultrasound upstream/downstream flow sampling sequence of the flowmeter is replaced with unidirectional signal transmission passing through the probe. Thus, the flowmeter calculates blood volume flow from two identical measurements resulting in a zero flow output regardless of the actual blood flow in the vessel. The blood flow recording mode was turned on to reestablish the actual flow measurement, and the distal aorta was mechanically occluded just above the bifurcation leading to true zero flow in the vessel. This true zero blood flow reading was compared to the zero blood flow measurement of the flowmeter to ensure the validity of the zero calibration system. 
Figure 1 - In vitro calibration of 1-mm (upper panel) and 2-mm (lower panel) probes implanted around the rat common carotid artery. Symbols represent experimental data obtained with 1 $\mathrm{mm}(\mathrm{N}=4)$ and 2-mm ( $=2)$ probes. The solid line represents the slope of the data and the dotted line, the line of identity. Parameters of linear regression analysis are shown.
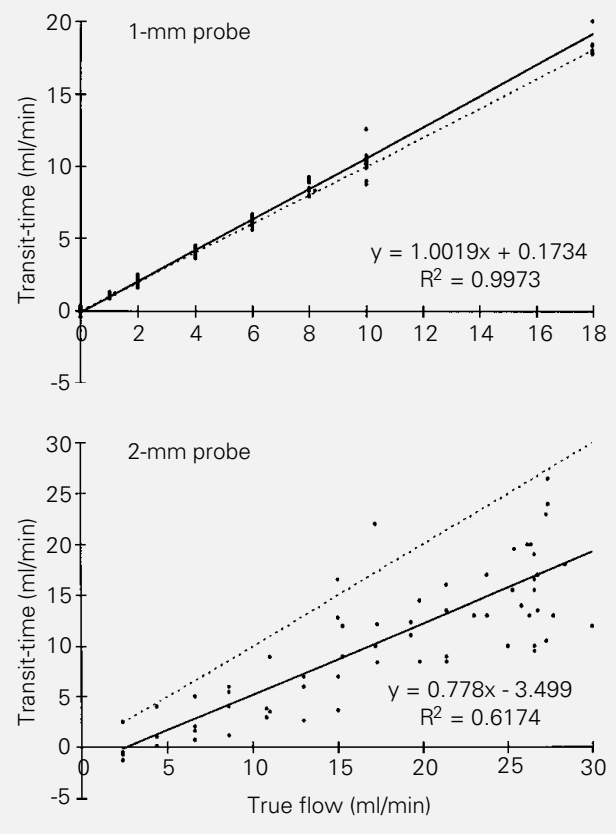

Other groups of rats chronically implanted with a 1-mm probe in the iliac artery (group II, $\mathrm{N}=7$ ) or in the renal artery (group III, $\mathrm{N}$ = 13) were tested for regional blood flow changes during exercise. Before probe implantation the rats were adapted to 5-min treadmill running episodes of 0.3 to $0.8 \mathrm{~km} /$ $\mathrm{h}, 0 \%$ degree for one week. The regional flow during rest, running and recovery was recorded on 3 successive days: on the first and second recording sessions, held 24 and $48 \mathrm{~h}$ after probe implantation, blood flow and heart rate were measured continuously. After stabilization of the variables, the rat ran on a treadmill (Inbramed, Porto Alegre, RS, Brazil) adapted for rats. The exercise test consisted of 4 min of graded exercise: 2 $\min$ at $0.4 \mathrm{~km} / \mathrm{h}$ and $2 \min$ at $0.8 \mathrm{~km} / \mathrm{h}, 0 \%$ degree. On the second day the rats were implanted with the arterial cannula into the contralateral femoral artery and the running protocol was repeated $24 \mathrm{~h}$ later. To assess the reproducibility of IF, arterial pressure (AP) and HR changes during exercise, 2 sessions of running episodes were held for each rat, 40-60 min apart. Before the running protocol, IF, HR and AP were recorded for
15 to $30 \mathrm{~min}$ to permit stabilization of the variables and to obtain true resting values.

Group IV consisted of 8 rats with a 2-mm probe (model 2SB) chronically implanted around the iliac artery. IF records were repeated on 3 consecutive days (40-60 $\mathrm{min}$ experimental sessions) in freely moving rats.

\section{Statistical analysis}

Data are reported as mean \pm SEM. The differences between groups were evaluated by the paired $t$-test or by analysis of variance for repeated measurements as appropriate. The level of significance was set at $\mathrm{P}<0.05$.

\section{Results}

Standardization of 1-mm and 2-mm transittime probes: in vitro validation and in vivo stability

Figure 1 provides the pooled data for relationships between true flow rate and measured flow with both probe sizes in the excised carotid artery. Under these experimental conditions the slopes for the 1-mm and 2-mm probes were quite different: 1.002 with a correlation coefficient of 0.997 , and 0.778 with a correlation coefficient of 0.617 for probes $1 \mathrm{R}$ and $2 \mathrm{~S}$, respectively. Although data of carotid flow obtained with the 1-mm flow probe were superimposed to the line of identity, those obtained with the $2-\mathrm{mm}$ probe were not. These results indicate that flow in the common carotid and iliac arteries is better quantitated by the 1-mm flow probe.

Iliac flow measurements after chronic implantation presented excellent stability. As observed in Figure 2 (upper panel for one conscious rat), both baseline HR and resting IF (pulsatile and mean) were stable and closely similar from day 2 up to day 6 after implantation. For this group, mean IF was on average $10.43 \pm 0.99 \mathrm{ml} / \mathrm{min}$, a value that was almost constant throughout the experimental period (Figure 2, lower panel). At 
rest, HR oscillated between $367 \pm 15$ and $398 \pm 24 \mathrm{bpm}$ during the 6 days of the experimental period. On the 6th day basal mean arterial pressure (MAP) was $110 \pm 3$ mmHg. It should be noted that at that time (after catheterization of the contralateral femoral artery) mean resting IF was $10.10 \pm$ $0.7 \mathrm{ml} / \mathrm{min}$. In contrast, the IF of conscious rats at rest differed markedly from the IF measured during surgery after acute implantation of the probe into pentobarbital-anesthetized rats. As illustrated in Figure 2, the IF of the same rats $(4.46 \pm 0.56 \mathrm{ml} / \mathrm{min})$ was significantly reduced by $50-60 \%$ during anesthesia when compared to the conscious state.

\section{Accuracy of probe measurements}

The sensitivity of the transit-time probe in reproducing physiological changes of IF was analyzed in chronically implanted rats also instrumented with an arterial catheter. As shown in Figure 3, IF records reproduced not only tonic but also phasic instantaneous absolute changes of pulsatile and mean flow, i.e., all the physiological patterns of flow changes such as increases/decreases simultaneous to elevations/falls in pressure (panels A and B), no changes regardless of pressure changes (panel C) and phasic flow oscillations opposite to pressure oscillations (panel D) were observed. Maintenance of elevated flow (tonic effect) simultaneously to pressure oscillation was also observed (panel E). Most of these flow patterns were presented by the 10 freely moving rats of group I. The flow changes related or not to pressure modification seemed to depend on the behavioral activities and demonstrated that the probe was able to detect with accuracy all patterns of IF changes.

\section{Zero flow baseline measurement}

The accuracy of flow measurements was tested in situ after median laparotomy and

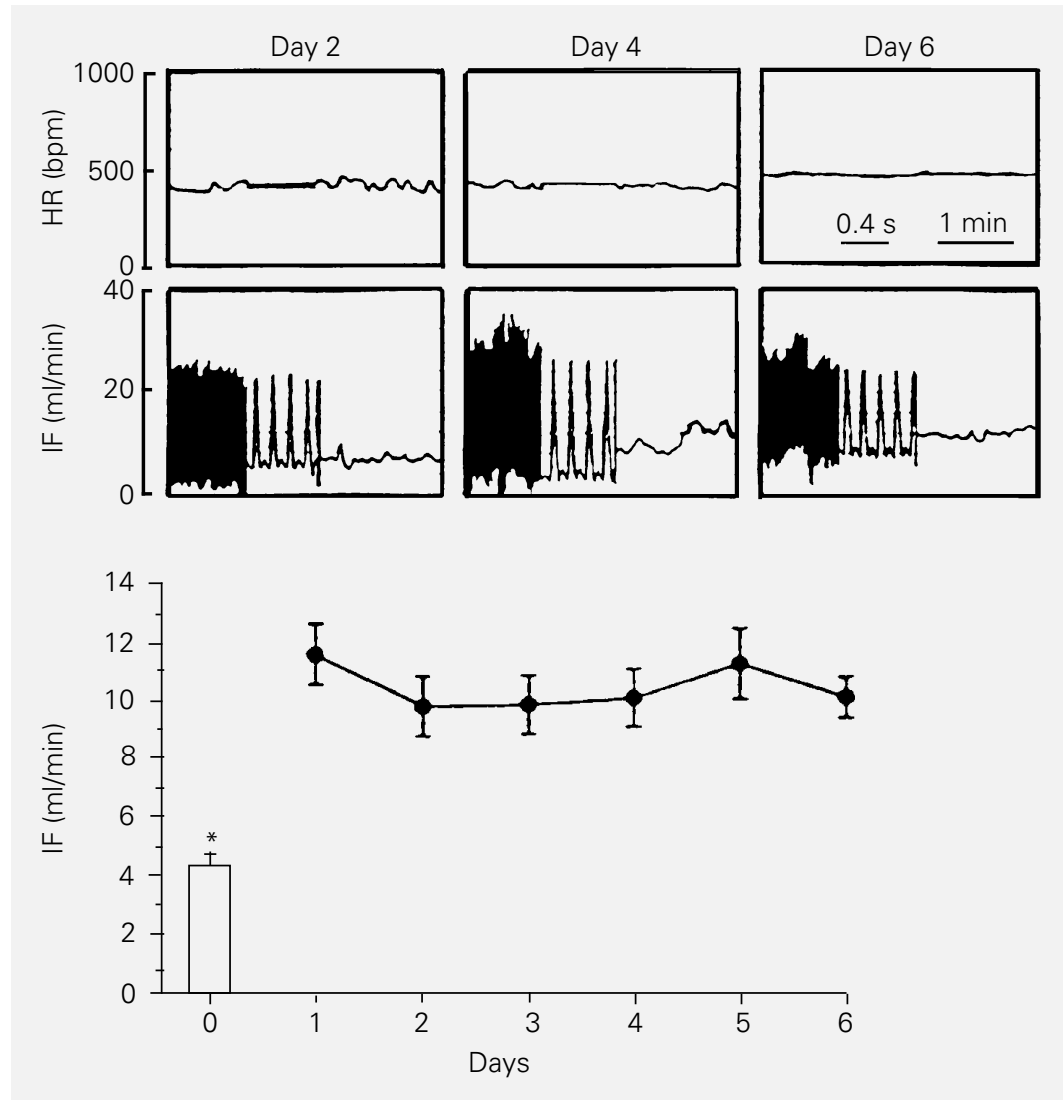

Figure 2 - Upper panel, Sequential recordings of heart rate (HR) and pulsatile and mean iliac flow (IF) at rest in a conscious rat with a perivascular 1-mm probe chronically implanted around the iliac artery. Lower panel, Mean values of resting iliac flow obtained on 6 consecutive days in the group of 10 rats. The bar represents the flow of the same rats immediately after probe implantation and during pentobarbital anesthesia. ${ }^{*} \mathrm{P}<0.05 \mathrm{com}$ pared to the conscious state (paired $t$-test).

exposition of the distal abdominal aorta and iliac artery implanted with the probe (ether anesthesia). Figure 4 shows a typical recording with the 1-mm probe taken from 2 rats. Brief occlusions (20 to $60 \mathrm{~s}$, panels B and C, respectively) actually caused zero pressure and true zero flow in the iliac artery which were properly detected by the probe. Mean flow measured after abdominal aortic occlusion in the group of rats as a whole was 0.33 $\pm 1.3 \mathrm{ml} / \mathrm{min}$, which was not different from zero. Also indicative of probe accuracy in detecting flow changes was the observation of the transient increase that characterizes the reactive hyperemic response after 1-min occlusion of the abdominal aorta (Figure 4, 
Figure 3 - Records of pulsatile (AP) and mean arterial pressure (MAP), heart rate (HR) and mean or pulsatile iliac flow (IF) in freely moving rats with a 1-mm probe chronically implanted around the iliac artery. See text for explanation.

Figure 4 - Simultaneous recordings of arterial pressure (AP, MAP) and mean iliac flow (IF) in ether-anesthetized rats during flowmeter zero flow measurements without interruption of blood flow (A) and the true zero flow measurements ( $B$ and $C$ ). panel C). Moreover, the flowmeter zero flow function (Figure 4, panel A) produced zero flow recordings similar to true zero flow measurements (panels B and C). Since no differences between the two methods for measuring zero blood flow were observed, the zero baseline function was used for calibration during the chronic experiments. We
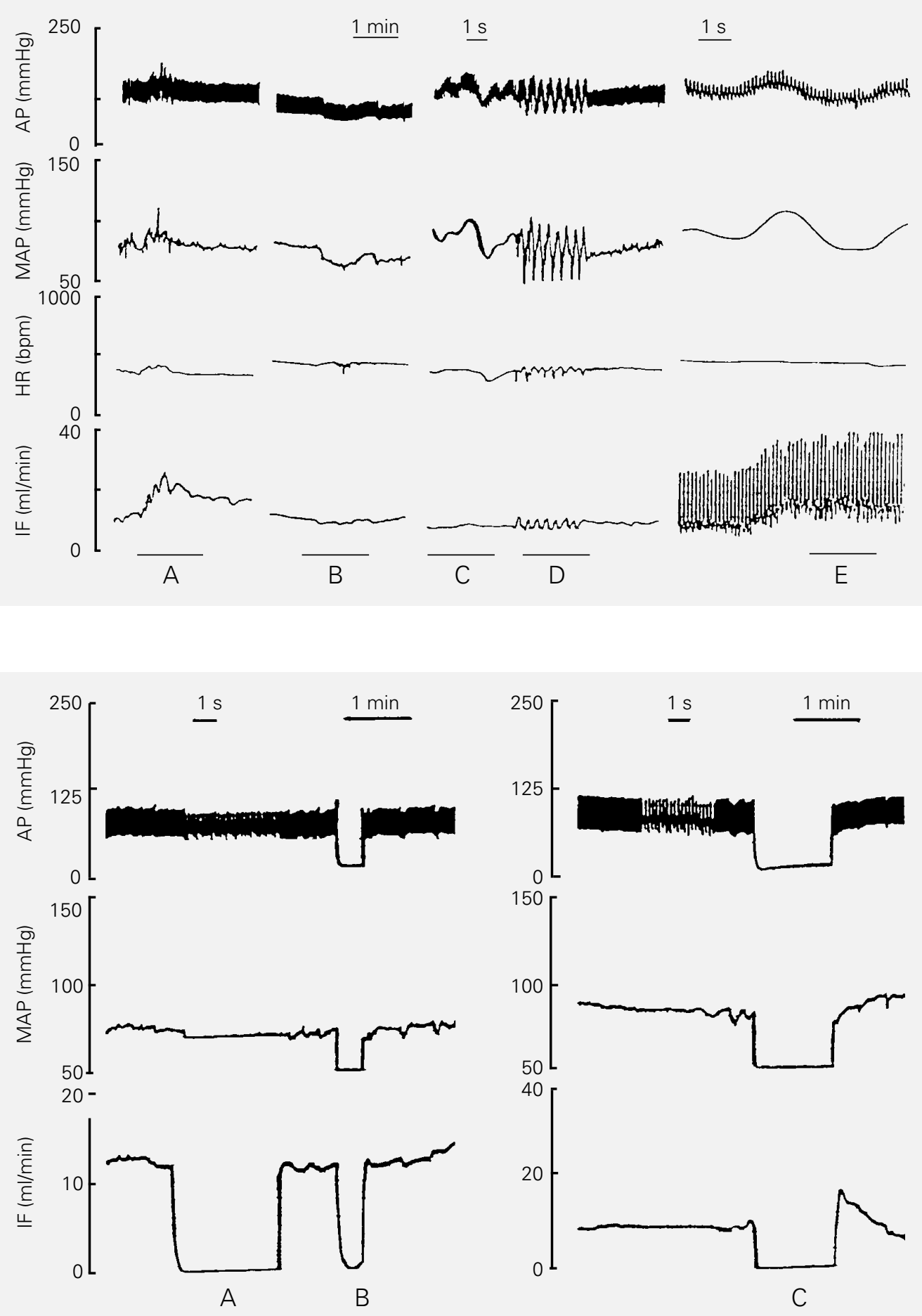


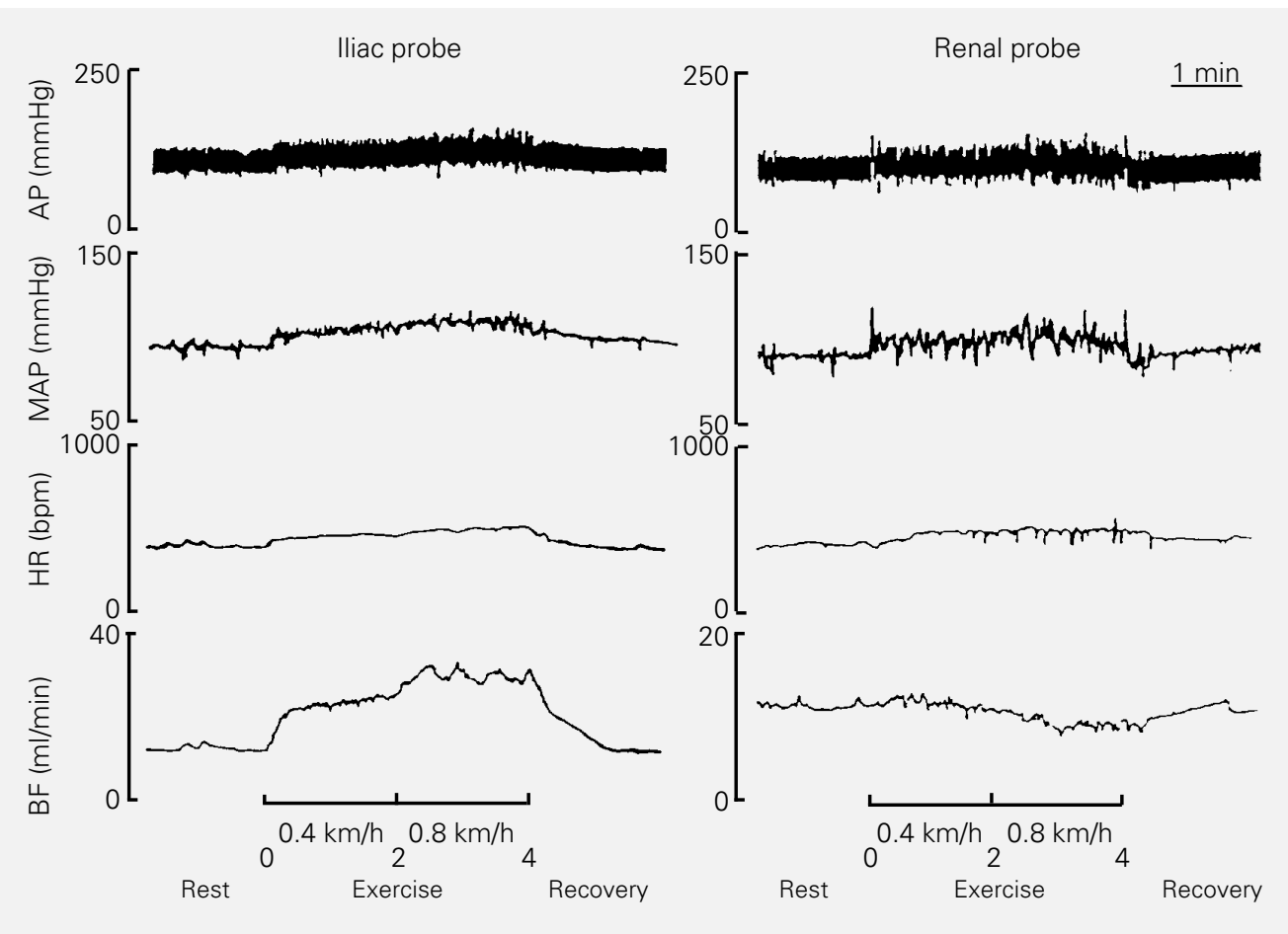

also confirmed true zero flow in some animals by measuring IF in situ after sacrifice at the end of the experiments.

\section{Regional flow changes during exercise}

The behavior of IF in response to dynamic exercise was tested simultaneously with AP records in another group of chronically instrumented rats during short 4-min runs on a treadmill. As illustrated in Figure 5, AP showed a small but similar increase $(+10$ to $+15 \mathrm{mmHg}$ ) during the running period in rats implanted with iliac or renal probes. HR and IF increased quickly during the first $15-30 \mathrm{~s}$ of mild exercise $(0.4 \mathrm{~km} / \mathrm{h})$, with an additional increase being observed at the beginning of moderate exercise intensity $(0.8 \mathrm{~km} /$ $\mathrm{h}$ ), and remained at this level until the end of the exercise. In contrast, renal flow (RF) showed opposite and smaller changes, i.e., it was almost unchanged at the beginning of exercise and showed a slow and continuous decrease until the end of the exercise protocol.
Table 1 - Resting mean arterial pressure (MAP), heart rate (HR), and iliac and renal flow measured with a $1-\mathrm{mm}$ probe in 2 groups of rats.

\begin{tabular}{lcc}
\hline & $\begin{array}{c}\text { \|liac flow } \\
(\mathrm{N}=7)\end{array}$ & $\begin{array}{c}\text { Renal flow } \\
(\mathrm{N}=10)\end{array}$ \\
\hline MAP (mmHg) & $109 \pm 3$ & $110 \pm 4$ \\
$\mathrm{HR}(\mathrm{bpm})$ & $370 \pm 6$ & $388 \pm 21$ \\
Absolute flow (ml/min) & $12.03 \pm 1.1$ & $10.86 \pm 1.7$ \\
Relative flow (ml min & $2.78 \pm 0.3$ & $3.04 \pm 0.5$ \\
$\left.100 \mathrm{~g} \mathrm{body} \mathrm{weight}^{-1}\right)$ & & \\
Flow index & 1.7 & 7.9 \\
(ml min &
\end{tabular}

Table 1 shows the baseline values of MAP, HR and regional blood flow obtained for the iliac and renal groups (protocols II and III). Baseline MAP, HR and absolute iliac and renal flow were similar for both groups. The renal group also had a smaller body weight ( $366 \pm 17$ vs $458 \pm 18 \mathrm{~g}$ in the iliac group) with a similar relative flow (3.04 \pm 0.5 vs $\left.2.78 \pm 0.3 \mathrm{ml} \mathrm{min}^{-1} 100 \mathrm{~g}^{-1}\right)$. On the other hand, calculations of flow index by correcting for kidney and hindlimb muscle
Figure 5 - Recordings of pulsatile (AP) and mean arterial pressure (MAP), heart rate (HR) and mean regional flow (BF) for 2 rats with a chronically implanted 1-mm probe (left panel, iliac flow and right panel, renal flow) during rest, exercise of mild $(0.4$ $\mathrm{km} / \mathrm{h})$ to moderate $(0.8 \mathrm{~km} / \mathrm{h})$ intensity (black bars) and recovery. 
Figure 6 - Time-course changes of absolute regional flow (BF) (iliac, $N=7$ and renal, $N=10$ ) during 4 min of exercise (black bar) and during recovery.

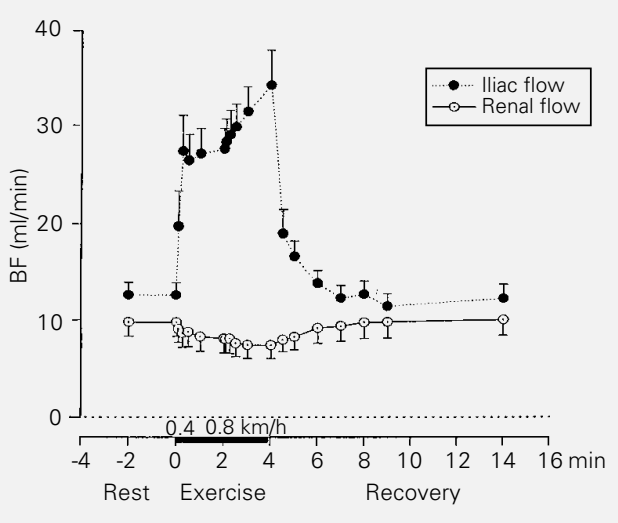

Table 2 - Comparison of mean arterial pressure (MAP), heart rate (HR) and iliac flow (IF) values in 2 running tests involving 7 normotensive rats.

Resting values, maximal changes at 2 different intensities and recovery values are presented.

\begin{tabular}{lcc}
\hline & 1st Running test & 2nd Running test \\
\hline MAP (mmHg) & & \\
Rest & $109 \pm 3$ & $112 \pm 3$ \\
$0.4 \mathrm{~km} / \mathrm{h}$ & $122 \pm 4$ & $122 \pm 3$ \\
$0.8 \mathrm{~km} / \mathrm{h}$ & $128 \pm 5$ & $125 \pm 4$ \\
$5-\mathrm{min}$ recovery & $114 \pm 4$ & $113 \pm 4$ \\
HR (bpm) & & \\
Rest & $370 \pm 6$ & $367 \pm 3$ \\
$0.4 \mathrm{~km} / \mathrm{h}$ & $431 \pm 8$ & $457 \pm 11$ \\
$0.8 \mathrm{~km} / \mathrm{h}$ & $491 \pm 3$ & $492 \pm 7$ \\
$5-\mathrm{min} \mathrm{recovery}$ & $371 \pm 12$ & $371 \pm 14$ \\
IF (ml/min) & & \\
Rest & & \\
$0.4 \mathrm{~km} / \mathrm{h}$ & $27.27 \pm 2.4$ & $27.11 \pm 2.53$ \\
$0.8 \mathrm{~km} / \mathrm{h}$ & $32.96 \pm 3.2$ & $32.14 \pm 3.5$ \\
$5-\mathrm{min} \mathrm{recovery}$ & $11.61 \pm 1.4$ & $11.00 \pm 1.02$ \\
& &
\end{tabular}

weight (on average $1.37 \mathrm{~g}$ and $7.49 \mathrm{~g}$, respectively) showed a higher value for renal tissue (7.93 vs 1.7, renal and hindlimb index, respectively).

The flow changes during exercise for both groups are illustrated in Figure 6. During mild exercise the first workload mean IF was increased by $+15.1 \pm 3.1 \mathrm{ml} / \mathrm{min}$, a value that is more than double (114\% increase) the resting IF, reaching $+20.43 \pm 2.45 \mathrm{ml} / \mathrm{min}$ $(+158 \%)$ at the second workload. At the same exercise intensity, RF showed minor changes compared to baseline, i.e., RF decreased on average $2 \mathrm{ml} / \mathrm{min}(-18 \%)$ during the second minute of $0.4 \mathrm{~km} / \mathrm{h}$ and $3-4 \mathrm{ml} /$ min at $0.8 \mathrm{~km} / \mathrm{h}(-25 \%$ below the resting value). As observed in Figure 6, the recovery of both IF and RF was progressive, reaching basal values 3-4 min after the end of exercise.

IF changes during acute exercise were reproducible. In two successive runs on a treadmill 40-60 min apart, similar AP, HR and IF responses were observed (Table 2): for the same AP changes, IF was increased 2.1 and 2.6 times for 0.4 and $0.8 \mathrm{~km} / \mathrm{h}$ in the first test and 2.1 and 2.5 times in the second test and a complete recovery of baseline values occurred 5 min after the end of exercise.

\section{Absolute flow measurements: 1-mm vs 2-mm probes}

An important and unexpected observation concerning absolute blood flow values is illustrated in Figure 7. Comparing IF values obtained with the 1-mm probe (group I) with the values presented by another group of rats chronically implanted with the 2-mm probe (group IV, $\mathrm{N}=8$ ), we observed that probes of different sizes showed different absolute IF values for the same condition, i.e., conscious state at rest. The values obtained with the large probe were significantly lower (57\% on average) than those observed with the 1-mm probe. It should be noted that the average value for the 3-day period $(4.45 \pm 0.19 \mathrm{ml} / \mathrm{min})$ was similar to that observed in vitro for a true flow of 10 $\mathrm{ml} /$ min (Figure 1).

\section{Postmortem findings}

No macroscopic evidence of iliac or renal artery damage was found in any group of rats instrumented with the transit-time flow probes. There was also no evidence of disturbance of hindlimb or renal blood flow 
since the performance of rats on the treadmill after probe implantation was similar to that observed 1-2 days before surgery.

\section{Discussion}

In the present study we demonstrate for the first time that a transit-time probe permits sequential and accurate measurement of regional blood flow responses during rest, exercise and recovery. In addition, we showed that after chronic implantation the technique provided stable and reliable measurements, thus permitting continuous (and simultaneously to pressure) recording of tonic flow as well as its phasic changes. In contrast to previous reports that blood flow is independent of the size of the transit-time probe $(14,15)$, we showed that $1-\mathrm{mm}$ and $2-\mathrm{mm}$ probes gave different absolute flow values, both in vitro and when chronically implanted, indicating that volumetric flow measurements are limited by the window of accuracy, which varies with probe size.

The transit-time technology for measuring dynamic changes of regional blood flow has several advantages over other methods such as electromagnetic, microsphere and Doppler system flowmetry: 1) small probe size permitting easy implantation; 2) continuous measurement of absolute blood flow representing an enormous advantage over phasic (microspheres, dye dilution) $(3,22,23)$ or relative measurements $(3,4,8-10) ; 3)$ probes are nonconstrictive and therefore do not interfere with the pulse pressure or percentage of diastolic flow as electromagnetic devices do $(7,14) ; 4)$ the probes are suitable for long-term studies (17) since the quality of the received signal improves with reactive infiltrating tissue growth around the probe/ vessel, providing the necessary acoustic conduction $(3,15)$, and 5) the flowmeter zero offset function permits frequent calibration in conscious freely moving rats. The perivascular transit-time probe has been successfully used for continuous assessment of flow

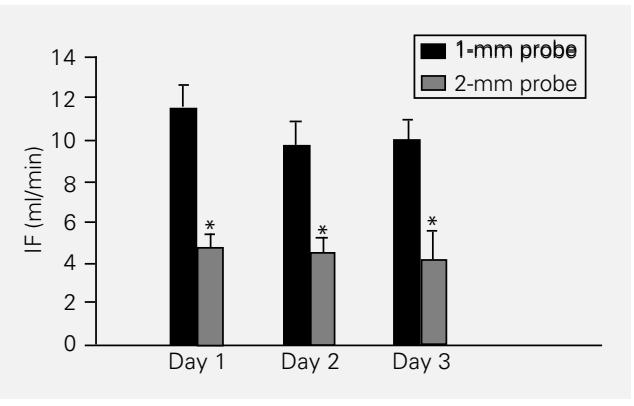

Figure 7 - Comparison of absolute iliac flow values (IF) obtained in the conscious state at rest (day 1 up to day 3 ) for rats implanted with a 1-mm (group I, $\mathrm{N}=10$ ) or 2-mm probe (group $\mathrm{IV}, \mathrm{N}=8) .{ }^{*} \mathrm{P}<0.05$ compared to the $1-\mathrm{mm}$ probe (ANOVA).

in coronary (17), mesenteric (13,15-17), renal $(13,14,17,24)$, carotid (13), gastric $(11,12)$, hindlimb $(13,17,25,26)$ and pulmonary arteries (7) and also for measurement of cardiac output $(3,8,13,15,24)$. Most of these studies, however, were carried out after acute implantation and few were conducted after chronic implantation. Chronic implantation, however, was employed to study drug effects on cardiac output and regional flows (8) and did not attempt to describe the daily flow profile of resting animals $(17,25)$. The results of the present experiments showed that implantable transit-time probes provided stable resting iliac flow values throughout the experimental period of 1 to 6 days. Another important result was the fact that the mean iliac flow of awake rats was not affected by arterial catheter placement in the contralateral femoral artery. These observations indicate that perivascular transit-time probes are suitable for chronic flow/pressure studies from the first day after implantation. In addition, the flow records obtained simultaneously to blood pressure records in awake rats demonstrated that transit-time probes reliably reproduce different flow patterns (mean and/or pulsatile) conditioned by pressure oscillations, opposite to pressure changes or bearing no relationship to them. Brief pulsatile records of femoral flow have already been reported for conscious rats (25), but they were obtained immediately before tail suspension and did not represent the true baseline flow. Bednarik and May (17) also employed this technique to measure mesenteric, renal, iliac and coronary blood flows 
simultaneously to pressure records in conscious sheep 16 months after probe implantation, but no time-course plots for resting flow values were presented. These observations, taken together with the present data, indicate that transit-time flowmetry is an effective and accurate technique for the quantitation of absolute baseline flow values over time. It is better than electromagnetic devices $(1,2)$ because it is nonconstrictive and does not show drift of the flow signal (6). The present protocol also included comparisons of zero blood flow obtained by the zero flow baseline function of the flowmeter and by measurement of zero blood flow after complete occlusion of the vessel. The two procedures provided similar measurements and, since there were no detectable changes in zero baseline function during the experimental protocol, we confirmed the advantage of the ultrasonic method over electromagnetic flowmetry in chronic studies. These observations agree with other studies (13, 15,17).

An important and unexpected observation was the difference between the 1-mm and 2-mm probes concerning the resting iliac flow values of conscious rats. This discrepancy could be explained by the different windows of accuracy for the 1-mm and 2mm probes. D'Almeida et al. (15) reported that true flow for the 2-mm probe is identical to measured flow only for values of about 20 $\mathrm{ml} / \mathrm{min}$ (ranging from 10.4 to $45 \mathrm{ml} / \mathrm{min}$ ), with marked deviations occurring at smaller or larger flow values. Our data with the 2$\mathrm{mm}$ probe also showed deviations from true flow, but extending for all ranges of flow tested. On the other hand, our data with the 1-mm probe indicated a perfect correlation between measured and true flows, confirming previous observations (15) and strongly suggesting that the flows measured with the $1-\mathrm{mm}$ probe accurately represent the true flow rates. Based on these observations, we suggest that the resting iliac flow of awake normotensive rats is about $2.8 \mathrm{ml} \mathrm{min} \mathrm{mi}^{-1} 100$ g body weight ${ }^{-1}$ or $1.7 \mathrm{ml} \mathrm{min}^{-1} \mathrm{~g}$ tissue $\mathrm{e}^{-1}$.

We also showed that measurements in conscious and anesthetized rats yielded different values even when using the same probe. It is well known that pentobarbital anesthesia depresses respiratory function, decreases sympathetic vasoconstrictor tone and increases vasodilator tone, thus reducing stroke volume, central venous pressure and arterial pressure (27). Accordingly, during anesthesia the iliac flow was proportionally decreased when compared to the conscious state. In keeping with these observations, care should be taken when comparing flow profiles obtained with different transit-time probe sizes during different states (conscious $v s$ anesthetized) and by different methods. Our absolute values are also in agreement with those obtained by Iriuchijima (1) who found a resting terminal aortic flow of $5.8 \mathrm{ml}$ $\min ^{-1} 100 \mathrm{~g} \mathrm{~g}^{-1}$, a value similar to that obtained here for iliac flow if we consider that aortic flow is shared by each iliac artery. It should be noted that the flow index calculated from the present data confirmed the well-known concept of flow distribution, i.e., that the renal circulation receives a larger portion of cardiac output than the skeletal muscle circulation under resting conditions.

One important observation is that the perivascular probe is an excellent method for measuring the dynamic changes in regional blood flow during exercise and recovery. It permits detection of instantaneous and continuous changes of mean or pulsatile flow profile, reproducing the actual flow pattern of different territories such as iliac (marked increase) and renal arteries (small decrease) in response to mild to moderate exercise intensity. The very rapid and pronounced increase of iliac flow was determined by withdrawal of sympathetic tone at the beginning of exercise, associated with local metabolic factors whose accumulation was proportional to the exercise intensity (28). On the other hand, the small reduction of renal flow during exercise was not unex- 
pected due to autoregulatory responses (29). Whether renal flow is maintained or decreased during exercise remains controversial (30). Most evidence suggests that redistribution of renal flow to exercising muscles only occurs when the exercise intensity is sufficiently severe and/or when a decrease in oxygen delivery to working muscles is imposed (30). Dynamic regional flow patterns during exercise have not been studied previously mainly due to the lack of an appropriate technique. Blood flow distribution within and among muscles in rats (18-21) and dogs (30) during exercise was determined by means of radiolabeled and radioactive microsphere techniques which permit few and static flow measurements, but do not provide information about the dynamic moment-to-moment flow changes as obtained here with the perivascular ultrasonic probe.
Based on the present observations, we propose that the transit-time technique is adequate to reproduce with accuracy any flow profile independent of absolute change (increase/decrease) and time-course (instantaneous or phasic/maintained or tonic).

In summary, the transit-time flow probe provides important advantages over other systems for the measurement of regional blood flow. It provides direct flow volume measurements, has a negligible zero offset and presents a long in vivo life span, thus permitting accurate measurements of regional flow after chronic implantation over long periods. It is a good and reliable method to measure instantaneously and continuously the dynamic changes of blood flow during rest, exercise and recovery in small animals such as rats.

\section{References}

1. Iriuchijima J (1983). Regional blood flow in conscious spontaneously hypertensive rats. Japanese Journal of Physiology, 33: 44-51.

2. lida $N$ (1995). Different flow regulation mechanisms between celiac and mesenteric vascular beds in conscious rats. $\mathrm{Hy}$ pertension, 25: 260-265.

3. Hartman JC, Olszanski DA, Hullinger TG \& Brunden MN (1994). In vivo validation of a transit time ultrasonic volume flow meter. Journal of Pharmacological Methods, 31: 153-160.

4. Janssen BJ, Oosting J, Slaaf DW, Persson PB \& Struijker-Boudier HA (1995). Hemodynamic basis of oscillations in systemic arterial pressure in conscious rats. American Journal of Physiology, 269: H62-H71.

5. Grohs JG, Huber S \& Raberger G (1993). Simultaneous assessment of cardiac output with pulsed Doppler and electromagnetic flowmeters during cardiac stimulation. Journal of Pharmacological and Toxicological Methods, 30: 33-38.

6. Gorewit RC, Aramondo MC \& Bristol DC (1989). Measuring bovine mammary gland blood flow using a transit-time ultrasonic flow probe. Journal of Dairy Science, 72: 116-119
7. Grant BJB, Paradowski LJ \& Fitzpatrick JM (1988). Effect of perivascular electromagnetic flow probes on pulmonary hemodynamics. Journal of Applied Physiology, 65: 1885-1890.

8. Dundore RL, Habeeb PG, Pratt PF, Becker LT, Clas DM \& Buchholz RA (1992). Differential hemodynamic responses to selective inhibitors of cyclic nucleotide phosphodiesterases in conscious rats. Journal of Cardiovascular Pharmacology, 19: 937944.

9. Hartley CJ \& Cole JS (1974). An ultrasonic pulsed Doppler system for measuring blood flow in small vessels. Journal of Applied Physiology, 37: 626-629.

10. Gelsema AJ, Copeland NE, Drolet G \& Bachelard H (1993). Cardiovascular effects of neural activation of the extended amygdala in rats. Brain Research, 626: 156-166.

11. Holzer $P$, Lippe ITh, Jocic M, Wachter Ch, Erb R \& Heinemann A (1993). Nitric oxidedependent and -independent hyperaemia due to calcitonin gene-related peptide in the rat stomach. British Journal of Pharmacology, 110: 404-410.
12. Holzer $P$, Wachter $C h$, Jocic $M$ \& Heinemann A (1994). Vascular bed-dependent roles of the peptide CGRP and nitric oxide in acid-evoked hyperaemia of the rat stomach. Journal of Physiology, 480: 575-585

13. Hoffman A, Grossman E, Öhman KP Marks E \& Keiser R (1989). Endothelin induces an initial increase in cardiac output associated with selective vasodilation in rats. Life Sciences, 45: 249-255.

14. Welch WJ, Deng X, Snellen H \& Wilcox C (1995). Validation of miniature ultrasonic transit-time flow probes for measurement of renal blood flow in rats. American Journal of Physiology, 268 (Renal, Fluid and Electrolyte Physiology, 37): F175- F178.

15. D'Almeida MS, Gaudin C \& Lebrec D (1995). Validation of 1- and 2-mm transittime ultrasound flow probes on mesenteric and aorta of rats. American Journal of Physiology, 268 (Heart and Circulatory Physiology, 37): H1368- H1372.

16. Barnes RJ, Comline RS, Dobson A \& Drost CJ (1983). An implantable transit time ultrasonic blood flow meter. Journal of Physiology, 345: 2P-3P. 
17. Bednarik JA \& May CN (1995). Evaluation of transit time system for the chronic measurement of blood flow in conscious sheep. Journal of Applied Physiology, 78: 524-530.

18. Laughling $\mathrm{MH}$, Armstrong RB, White J \& Rouk K (1982). A method for using microspheres to measure muscle blood flow in exercising rats. Journal of Applied Physiology, 52: 1629-1635.

19. Armstrong RB \& Laughling $\mathrm{MH}$ (1984). Exercise blood flow patterns within and among rat muscles after training. American Journal of Physiology, 246 (Heart and Circulatory Physiology, 15): H59-H68.

20. Peterson DF, Armstrong RB \& Laughling $\mathrm{MH}$ (1988). Sympathetic neural influences on muscle blood flow in rats during submaximal exercise. Journal of Applied Physiology, 65: 434-440.

21. Mush TI \& Terrel JA (1992). Skeletal muscle blood flow abnormalities in rats with chronic myocardial infarction: rest and exercise. American Journal of Physiology, 262 (Heart and Circulatory Physiology, 31): $\mathrm{H} 411-\mathrm{H} 419$.
22. Kobayashi N, Kobayashi K, Kouno K, Horinaka S \& Yagi S (1994). Effects of intra-arterial injection of colored microspheres on systemic hemodynamics and regional blood flow in rats. American Journal of Physiology, 266 (Heart and Circulatory Physiology, 35): $\mathrm{H} 1910-\mathrm{H} 1917$.

23. Kissling G, Ross C \& Brandle M (1993). Validity of thermal dilution technique for measurement of cardiac output in rats. American Journal of Physiology, 265 (Heart and Circulatory Physiology, 34): H1007-H1013.

24. Shiraichi $H$, Silverman NH \& Ruldolph AM (1993). Accuracy of right ventricular output estimated by Doppler echocardiography in the sheep fetus. American Journal of Obstetrics and Gynecology, 168: 947953.

25. Roer DR \& Dillaman M (1994). Decreased femoral arterial flow during simulated microgravity in the rat. Journal of Applied Physiology, 76: 2125-2129.
26. Wilkening RB, Boyle DW \& Meschia G (1988). Measurement of blood flow and oxygen consumption in the pelvic limb of fetal sheep. Proceedings of the Society for Experimental Biology and Medicine, 187: 498-505

27. Fragen RJ (1986). Cardiovascular effects of intravenous anesthetics. In: Altura BM \& Halevy S (Editors), Cardiovascular Actions of Anesthetics and Drugs Used in Anesthesia. Vol. 1. Karger, New York, 5173.

28. Mitchell JH (1990). Neural control of the circulation during exercise. Medicine and Science in Sports and Exercise, 22: 141154

29. Cowley Jr AW (1992). Long term control of arterial blood pressure. Physiological Reviews, 72: 231-300.

30. Mush TI, Friedman DB, Pitetti KH, Haidet GC, Stray-Gundersen J \& Mitchel JH (1992). Regional distribution of blood flow of dogs during graded dynamic exercise. Journal of Applied Physiology, 63: 22692277. 